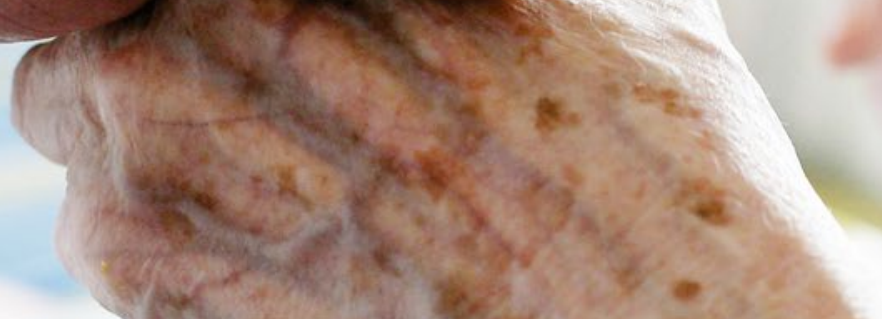

\title{
Beihilfe zum Suizid - Anmerkungen aus Schweizer Perspektive
}

Die Diskussion um dieses brisante Thema spiegelt in den Medien und in den politischen Diskussionen die ganze Bandbreite der Sichtweisen wider und ist auch nach den aktuellen politischen Entscheiden und dem Inkrafttreten von $\$ 217$ Strafgesetzbuch (StGB) nicht zu Ende.

Hier spielen politische, ethische, religiöse, familiäre, juristische und professionelle Erfahrungen mit hinein, die von unterschiedlicher Objektivität und unterschiedlichen kulturellen Einflüssen geprägt sind. Im Bundestag lagen vier unterschiedliche Entwürfe zur Diskussion und Entscheidung vor, die unter anderem im Deutschen Ärzteblatt dargelegt wurden [Klinkhammer G, Richter-Kuhlmann E. Dtsch Arztebl. 2015; 112(4): A-124/B-108/C-104].

\section{Ausreichende Linderung nicht immer möglich}

Nicht wenige der zahlreichen Wortführer haben vermutlich noch nie einen onkologischen Patienten in der späten palliativen Lebensphase betreut. So wird zuweilen vergessen, dass es durchaus Patienten gibt, deren Leiden auch durch onkologisch erfahrene Ärzte - onkologische Spezialisten, Palliativmediziner und Schmerztherapeuten - nicht entscheidend und für die $\mathrm{Pa}$ tienten zufriedenstellend gelindert werden kann [Smith TJ, Saiki CB. Mayo Clin Proc. 2015;90(10):1428-39]. Zweifelsohne wird leider nicht bei jedem Patienten und nicht immer rechtzeitig auf eine interdisziplinäre Abstimmung geachtet, sodass palliative Aspekte zu kurz kommen, zum Nachteil der Patienten [Smith TJ, Saiki CB. Mayo Clin Proc. 2015;90(10):1428-39]. Diese Unterversorgung sollte nicht zu einer frühzeitigen und womöglich unnötigen Diskussion um einen assistierten Suizid einzelner Patienten führen, sondern im Sinne der besseren Lebensqualität der Patienten und auch im Sinne einer längeren, vergleichsweise guten Lebensstrecke geführt werden [Temel JS et al. N Engl J Med. 2010;363(8):733-42].

Andererseits müssen Lösungen für Patienten gefunden werden, die unter nicht ausreichend behandelbaren Schmerzen leiden, vor allem in der präfinalen Phase; auch andere tumorbedingte Symptome, wie zunehmende neurologische Defizite, ausgeprägte Ängste, Störungen des Gastrointestinaltraktes bis hin zu Ileuszuständen, Einschränkungen der kardialen und pulmo-

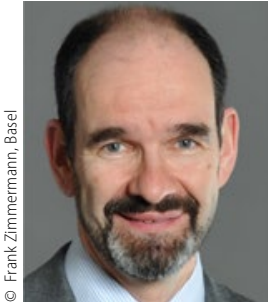

Prof. Dr. Frank Zimmermann

Klinik für Strahlentherapie und

Radioonkologie

Universitätsspital Basel, Schweiz

Frank.Zimmermann@usb.ch

nalen Leistungen mit hochgradiger Atemnot können unsere onkologischen Patienten beträchtlich belasten, daneben deutliche depressive Beschwerden [Janberidze E et al. BMJ Support Palliat Care. 2015 Feb 9. (Epub ahead of print)]. Aus eigener Erfahrung gelingen auch unter früher Einbindung psychoonkologischer Experten und Palliativmediziner sowie Schmerzspezialisten dennoch nicht bei jedem Patienten eine suffiziente Einstellung und eine ausreichende Linderung der genannten Beschwerden. Vor diesem Hintergrund erscheint mir die Einbindung einer Sterbehilfe aus Sicht der betroffenen Patienten durchaus nachvollziehbar, um ein unerträgliches Dasein auf eigenen Wunsch jederzeit beenden zu können.

\section{Strafrechtlich mehr Sicherheit in der Schweiz}

In Kenntnis der kontroversen medizinischen Betreuungsmöglichkeiten in Deutschland und in der Schweiz - unter Einbindung einer Palliativmedizin, einer Psychoonkologie, einer Schmerzambulanz und religiöser Angebote - hat sich für viele Patienten bereits das Wissen um die theoretische Möglichkeit einer Suizidbeihilfe als unterstützend und haltgebend herausgestellt. Dies wird nicht nur in Deutschland kontrovers diskutiert, sondern wurde es auch über lange Zeit in der Schweiz [Eidgenössisches Justiz- und Polizeidepartement 2009. Organisierte Suizidhilfe soll geregelt werden. Medienmitteilung vom 28. Oktober].

Unter der rechtlichen Regelung des Strafgesetzbuches bieten aktuell drei große Gesellschaften in der Schweiz als konfessio- 


\section{Hier steht eine Anzeige.}

Springer 
nell und politisch neutrale Institutionen Unterstützung beim Suizid an (Suizidbeihilfe): Exit Suisse Romande, Exit Deutsche Schweiz und Dignitas. Es handelt sich dabei um Vereine, die nach ihren Statuten weder Erwerbs- noch Selbsthilfezwecke verfolgen dürfen. Die durchaus relevanten Mitgliedsbeiträge eines Vereins werden laut dessen Statuten im Falle eines Finanzüberschusses in Maßnahmen zur Verhinderung von risikoreichen Suizidversuchen und zur Verminderung der Anzahl der Suizide investiert. Die anderen Vereine äußern sich nach meiner Kenntnis nicht zur Verwendung der Mitgliedsbeiträge. Aufgrund der Vorstandsbesetzungen und Überwachungen dürften aber selbstsüchtige Motive ausgeschlossen sein, es besteht jedoch eine Geschäftsmäßigkeit.

In den letzten Jahren zeigt sich eine beständige Zunahme der Suizidbeihilfen in der Schweiz: 1.070 Menschen begingen Suizid ohne Begleitung, 587 Schweizer Einwohner nahmen die Suizidbeihilfe in Anspruch. Dies sind unter 0,8\% sämtlicher Todesfälle in der Schweiz. Mit ca. 40 \% machen Krebspatienten einen großen Anteil aus. Hinzu kommen aus dem Ausland eingereiste schwerkranke Patienten (Epidemiologie von Suiziden, Suizidversuchen und assistierten Suiziden in der Schweiz, April 2015, unter www.bag.admin.ch). Diese Beihilfe ist entsprechend der Richtlinien zur Suizidbeihilfe keine ärztliche Tätigkeit. Sollten gar selbstsüchtige Motive hierbei verfolgt werden, droht ein Freiheitsentzug von bis zu 5 Jahren ( $\$ 115$ StGB). Die Begrenzung auf selbstsüchtige Motive im Gegensatz zur Geschäftsmäßigkeit ermöglicht jedoch den wiederholten Einsatz einer Sterbehilfe, während dies in Deutschland unter den Experten in der Auslegung diskutiert wird, da eine Geschäftsmäßigkeit vorgeworfen werden kann. Die Regelung in der Schweiz bietet somit für die Beteiligten strafrechtlich mehr Sicherheit.

\section{Entlastung der Patienten durch theoretische Möglichkeit der Suizidbeihilfe}

In den letzten Jahren konnte ich im Umgang mit meinen Patienten einige Erfahrungen sammeln, vor allem in Hinblick auf eine offene Gesprächsführung. Nicht wenige meiner Patienten waren bereits vor meiner Betreuung Mitglieder in einem der genannten Vereine zur Suizidbeihilfe, da nur Vereinsmitglieder deren Angebot nutzen können. Die Mehrzahl dieser Patienten hat die Angebote der Vereine jedoch nie genutzt; es gab ihnen aber Sicherheit und Halt, im Einzelfall auf die Angebote zurück- greifen zu können. Eindeutig war die Erleichterung sämtlicher Patienten, wenn das Thema von mir aktiv angesprochen wurde und wir ein offenes Gespräch über die vielfältigen Möglichkeiten der Palliativmedizin und der alternativen Wege führen konnten. Dies scheint nicht die Regel zu sein [Smith TJ, Hillner BE. N Engl J Med. 2011;364(21):2060-5] und auch nicht ganz einfach, denn wer fühlt sich berufen und fähig, solch ein $\mathrm{Ge}$ spräch zu führen und den betroffenen Patienten ausreichend gut zu kennen [Tolle SW et al. N Engl J Med. 2015;372(7):667-70]. In keinem Fall habe ich durch das Gespräch, in dem ich die Nutzung der Suizidbeihilfe nicht angeregt, sondern nur angesprochen habe, eine Entscheidung gegen sinnvolle lebensverlängernde Maßnahmen erlebt. Das Gespräch alleine, die Offenheit und die Nähe führen nicht selten zu einem besseren Verständnis füreinander und zu einer besseren Lebensqualität [Klinkhammer G, Richter-Kuhlmann E. Dtsch Arztebl. 2015;112(4):A-124/B108/C-104]. Eine Einbindung der Angehörigen in solche Gespräche erscheint mir dabei essentiell, um diese je nach der endgültigen Entscheidung der betroffenen Patienten frühzeitig auf eine Trennung vorbereiten zu können.

Man mag durchaus geteilter Meinung sein, ob eine Regelung über ein Vereinswesen sinnvoll ist. In jedem Fall sollten der Entscheidungsprozess zum und die Betreuung während des assistierten Suizids klar definiert sein. Hierzu könnten unabhängige juristische und medizinische Experten involviert werden.

Zu hinterfragen sind aus meiner Sicht allerdings Äußerungen auf parteipolitischen Internetseiten wie „bei der Sterbebegleitung müssen wir eine Begleitung bis in den Tod fördern ... das Leben und vor allem die Würde sind dem Menschen nicht disponibel“. Hierdurch könnte das Leiden mancher onkologischer Patienten akzeptabel werden und das wäre nicht sinnvoll. Ich kann nur wünschen, dass der $\$ 217$ StGB in seiner jetzigen Fassung [Bundesgesetzblatt Jahrgang 2015 Teil I Nr. 49] nicht dazu führt, dass neben der wünschenswerten und nötigen Unterbindung einer kommerziellen und gewinnorientierten Beihilfe unseren Patienten die Möglichkeit versagt wird, wirklich in Würde und nicht in großem Leid zu sterben!

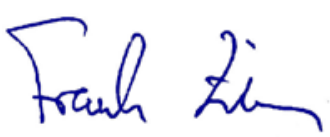

